\title{
Overall Quality Performance of Long-Term Care Homes in Ontario
}

Andrea Wilkinson, Vinita Haroun, Tommy Wong, Nancy Cooper and Mark Chignell

\begin{abstract}
In this paper, individually reported long-term care (LTC) quality indicators have been supplemented with the composite Qindex measure and applied to 614 homes in Ontario, Canada. This study (1) describes the overall quality performance of LTC homes across five years (2012-2017) and (2) determines if organizational factors impact quality performance. The results demonstrate significant, continuous sector-wide improvement in overall quality performance (as assessed by the Qindex) over time and significant differences in quality based on home size, operator size and ownership. This paper positions the Qindex, a global metric of quality, as a valuable tool for quality measurement and management in the LTC sector.
\end{abstract}

\section{Introduction}

\section{Long-term care in Ontario, Canada}

Long-term care (LTC) homes in Ontario are publicly funded facilities providing on-site nursing care and access to medical services 24 hours a day to residents. In Ontario, there are currently 625 licensed LTC homes that collectively care for approximately 100,000 residents annually (Ontario Long Term Care Association 2018). Over time, residents have entered LTC homes with more complex conditions and health needs, requiring increased levels of care. In addition, many residents have some form of cognitive impairment (approximately 90\%) and around $85 \%$ require extensive or complete support with their activities of daily living (CIHI 2016-2017).

Maintaining high quality of care in the LTC sector is critically important to residents, their family members, home operators, government and society as a whole. Because of the vulnerability of this group of citizens, it is vital to maintain public oversight on quality and safety through mandatory inspections and public reporting of quality indicators (QIs). We developed a standard overall indicator of quality that could be used to compare and manage quality across homes to provide public reassurance of an overall indication of quality performance in the LTC sector (Wilkinson et al. 2019). Using that measure of overall quality, this study has two objectives:

1. to describe trends in quality performance of LTC homes in Ontario, from 2012 to 2017, and

2. to determine if organizational factors (home size, operator size, ownership) have an impact on quality performance.

\section{Literature Review Approaches to accountability}

Accountability to governing bodies in healthcare (e.g., government, regional health authority) helps ensure that goals are being met and performance is being improved upon (Deber 2014; Denis 2014; Kromm et al. 2014). According to 
Deber (2014), there are several key approaches to accountability used in the healthcare system across Canada, including financial incentives, regulations and information (directed toward potential service users). Information, as an accountability driver, can be shared through report cards, inspection and incidence reports and QIs (Baker et al. 2004; CIHI 2017; Deber 2014; HQO n.d.). The implementation of nursing home report cards has been shown to lead to improvements in overall quality performance (Mukamel et al. 2008, 2015).

Over the past several years, the LTC sector in Ontario has gone through substantial changes to accountability, including to whom the sector is accountable and which performance targets are specified (Berta et al. 2014). In particular, accountability in the LTC sector in Ontario has substantially shifted away from historic accountability targets around operations to increasing the focus on quality improvement (Berta et al. 2014). The LTC sector in Ontario is also strictly regulated (Berta et al. 2014), and all homes are inspected routinely by the Ministry of Health and Long-Term Care (MOHLTC). The intended purpose of these inspections is to promote resident safety, quality of care and quality of life (Berta et al. 2014; Ontario MOHLTC 2008). Inspections are focused on measuring the performance of each home against compliance with the provincial legislation independent of its performance on the nine publicly reported QIs. Compliance is determined on the basis of three factors: the severity of the non-compliance, the scope of the non-compliance and the licensee's history of compliance with the legislation and any service accountability agreement (s.299(1), Long-Term Care Homes Act, Ontario Regulation 79/10: General, 2007). Failure to comply with legislation and regulation can result in a range of repercussions, from fines to revoked licences (Berta et al. 2014).

LTC homes in Ontario began to voluntarily publicly report on four QIs with the introduction of the provincial Residents First quality initiative in 2009 (Berta et al. 2014; HQO 2013b, 2015). Once the Resident Assessment Instrument Minimum Data Set (RAI-MDS) 2.0 was fully implemented across the sector, public reporting at the home level became mandatory (Berta et al. 2014; HQO 2013b, 2015). The Canadian Institute for Health Information (CIHI) introduced its public reporting website in 2015, extending public reporting on QIs to all LTC homes submitting data to CIHI from across the country (CIHI 2019a).

Since 2006, the 14 regional Local Health Integration Networks (LHINs) in Ontario (regional health authority responsible for administering, planning, integrating, coordinating and distributing public healthcare services and funding across the province) became responsible for managing accountability agreements (Berta et al. 2014; Eliasoph et al. 2007). In 2010, LTC homes in Ontario signed the new LHIN-sector service accountability agreements, bringing them under the umbrella of LHIN accountability. A map depicting the 14 LHIN divisions can be found online (http://www.lhins.on.ca).
At the time of publication, legislation to establish Ontario Health (Bill 74) has been tabled and a new board established, amalgamating the functions of the LHINs, Health Quality Ontario (HQO) and several other health agencies. This system change will likely involve modified ways of implementing accountability for the LTC sector.

\section{Quality outcomes and types of homes in long-term care} On an annual basis, Ontario LTC homes engage in a quality improvement cycle led by HQO. Until recently, HQO served as the province's advisor on quality, was responsible for monitoring and reporting on the quality of healthcare in Ontario and acted as an agent to continuously support quality improvement efforts using scientific evidence and rigour (HQO 2013a, 2014).

In addition, at a federal level, $\mathrm{CIHI}$ is mandated to deliver comparable and actionable information to accelerate improvements in healthcare, health system performance and population health across the continuum of care (CIHI 2016). Currently, under CIHI's interactive "Your Health System" tool, nine QIs are reported for LTC homes across Canada: experiencing pain, experiencing worsening pain, falls in the past 30 days, improved physical functioning, potentially inappropriate use of antipsychotics, restraint use, worsened depressive mood, worsened physical functioning and worsened pressure injuries - at the time of publication, the tool still refers to this indicator as "worsened pressure ulcers" (CIHI 2019b). The literature suggests that several home-defining characteristics (e.g., home size, operator size and ownership) have an impact on quality performance.

\section{Home size}

Current evidence regarding the impact of home size on quality should be interpreted with caution. According to Baldwin et al. (2017), smaller homes offer better quality of care and service to residents than larger homes. However, the studies reviewed by Baldwin et al. did not consistently report or calculate quality outcome measures. For reliable conclusions about quality performance to be drawn, quality should be systematically calculated and compared across studies.

\section{Ownership and operator size}

Regarding the impact of ownership on quality, the findings from the literature are inconsistent. This is primarily because of a lack of consensus in measuring quality. For example, are hospitalization and mortality rates appropriate proxies for quality performance? McGregor et al. (2006) found that, overall, for-profit homes had higher hospitalization rates (suggesting poorer quality of care) than not-for-profit homes, but there was no difference in mortality rates. The not-for-profit advantage found in the study by McGregor et al. (2006) may have been boosted by the fact that those 
homes were more likely to be attached to a hospital or amalgamated with other regional health authorities (i.e., multi-site), improving access to a broader range of services (e.g., nursing and physical professionals, geriatricians) and diagnostics (which may directly reduce hospitalization rates by screening out false alarms). Of note, McGregor et al. also showed that single-site, not-for-profit homes demonstrated higher hospitalization rates than for-profit homes (after adjusting for home size). In line with McGregor et al.'s initial findings, Tanuseputro et al. (2015) demonstrated that publicly funded private homes showed not only significantly higher rates of hospital admissions but also higher mortality rates than not-for-profit homes (including municipal homes). Hillmer et al.'s (2005) systematic review of 38 studies (37 American and one Canadian) found that across various quality outcomes (defined as, e.g., hospitalization, pressure ulcers, incontinence, dehydration), not-for-profit homes demonstrated enhanced quality compared to for-profit/private homes. One key issue in the aforementioned studies is the use of hospitalization and mortality rates as indicators of quality performance. CIHI reports nine QIs for LTC homes across Canada, none of which is hospitalization or mortality rate.

Assessing the situation in Ontario, this study describes trends in overall quality performance using a consistent measure across nine QIs and explores the impact of organizational factors, including home size, operator size and ownership, on overall quality performance.

\section{Methods}

\section{Data source}

The RAI-MDS version 2.0 is the standardized assessment tool for capturing the health status of LTC residents in Ontario as per the MOHLTC health data, including quality performance data for the nine QIs, and is placed onto CIHI's interactive tool (CIHI 2019b), where it can be downloaded for public use.

\section{The Qindex}

The Qindex is a composite QI score created by averaging the $\mathrm{z}$ scores of each of the nine QI variables over a five-year period: 2012/2013-2016/2017 (specifically, April 1, 2012 to March $31,2017)$. The $\mathrm{z}$ scores were calculated separately for each QI but on data aggregated over the five-year period so that yearon-year trends would be visible. For a more detailed description of the development of the composite quality score, see the article by Wilkinson et al. (2019).

\section{Sample size, inclusion criteria and exclusion criteria}

Data were available for 634 homes (2016-2017). However, 20 facilities were excluded because they were registered hospitals (often with short-stay LTC beds on very small units). As a result, the final sample consisted of a total of 614 LTC homes in Ontario.
For home characteristics, data were sourced from several different locations. For number of beds, data were captured through a mixture of the Ontario Long Term Care Association's (OLTCA) internal membership database and the ministry's public reporting website (Ontario MOHLTC 2019). Home size was then manually classified based on the number of beds. Operator size data were pulled from the OLTCA's internal membership database; therefore, only members of the OLTCA were included in the analyses and figure related to "operator size." Ownership designation was also collected from the ministry's public reporting website (as above).

When placing a home into a comparison category (e.g., small home, municipal ownership), the classification was based on specification from the most recent year (2016-2017). In this way, only the most up-to-date category assignment information was applied.

\section{Results}

In Table 1, the sample by home size, operator size and ownership across the past five years is shown. Of note, the proportions of each type of home (i.e., home size, operator size and ownership) were fairly stable over the five-year period.

\section{Overall Qindex}

Figure 1 shows the trend in overall Qindex performance over a five-year period. The results revealed that LTC homes improved quality of care steadily, encompassing an increase of approximately one-quarter of a standard deviation $(S D)$ unit over the five-year period. Repeated-measures analysis of variance (ANOVA) with Greenhouse-Geisser adjustment of the degrees of freedom (because of violation of the assumption of sphericity, e.g., Field et al. 2012, p. 554) showed a significant main effect of year, $F(3.004,1811.31)=136.99$, partial $\eta^{2}=.19, p<0.001$, with the line chart in Figure 1 showing overall improvement in QIs from 2012 to 2017.

Next, performance on the Qindex was explored across various home characteristics: home size, operator size and ownership. For each analysis, a 3 (home characteristic) $\times 5$ (year) mixed-model ANOVA was conducted with Qindex performance as the dependent variable. Each home characteristic was a between-subjects variable and year was a within-subjects variable. Home and operator size were categorized as small, medium or large, and ownership was private, not-for-profit or municipal. In all cases where Mauchly's test of sphericity was significant (indicating violation of the assumption of sphericity), the degrees of freedom were adjusted using the Greenhouse-Geisser criterion. Because the main effect of year was reported earlier in the discussion of Figure 1, in the remaining analyses, year will only be discussed in terms of its interaction with the home characteristic being evaluated. 
TABLE 1.

Overview of the sector: home size, operator size and ownership

\begin{tabular}{|c|c|c|c|c|c|}
\hline & 2012-13 & 2013-14 & 2014-15 & 2015-16 & 2016-17 \\
\hline \multicolumn{6}{|l|}{ Home size } \\
\hline Small (1-96 beds) & $235(39 \%)$ & 235 (39\%) & 235 (39\%) & $236(38 \%)$ & $236(38 \%)$ \\
\hline Medium (97-160 beds) & $229(38 \%)$ & $229(38 \%)$ & $231(38 \%)$ & $233(38 \%)$ & $233(38 \%)$ \\
\hline Large (+160 beds) & $140(23 \%)$ & $140(23 \%)$ & $143(23 \%)$ & $144(23 \%)$ & $145(24 \%)$ \\
\hline Total & 604 & 604 & 609 & 613 & 614 \\
\hline \multicolumn{6}{|l|}{ Operator size } \\
\hline Large (>20 homes) & $171(41 \%)$ & $171(41 \%)$ & $173(41 \%)$ & $173(41 \%)$ & $173(40 \%)$ \\
\hline Medium (4-20 homes) & $106(25 \%)$ & $106(25 \%)$ & $108(25 \%)$ & $109(26 \%)$ & $109(25 \%)$ \\
\hline Small (1-3 homes) & $143(34 \%)$ & $143(34 \%)$ & $144(34 \%)$ & $145(34 \%)$ & $146(34 \%)$ \\
\hline Total & 420 & 420 & 425 & 427 & 428 \\
\hline \multicolumn{6}{|l|}{ Ownership } \\
\hline Private & $347(57 \%)$ & 347 (57\%) & $352(58 \%)$ & $354(58 \%)$ & $355(58 \%)$ \\
\hline Not-for-profit & $156(26 \%)$ & $156(26 \%)$ & $156(26 \%)$ & $158(26 \%)$ & $158(26 \%)$ \\
\hline Municipal & $101(17 \%)$ & $101(17 \%)$ & $101(17 \%)$ & $101(16 \%)$ & $101(16 \%)$ \\
\hline Total & 604 & 604 & 609 & 613 & 614 \\
\hline
\end{tabular}

Note: Number of homes per a given year that fit into each characteristic denoted. Percentage of total represented in parentheses. Of the Ontario Long Term Care Association (OLTCA) membership, 69\% of small homes are licensed to operators of two or more homes and/or co-located on campuses with retirement or assisted living apartments. Some home characteristics (i.e., operator size) were only known for members of the OLTCA; thus, the sample size data related to this factor were limited to the OLTCA membership. The sample of homes with calculated Qindex scores varied across years. The variation in sample size across the years is because of several factors (e.g., new homes that did not collect quality data in previous years)

\section{FIGURE 1.}

Overall quality performance (as indexed by the Qindex) across five years (2012-2017)

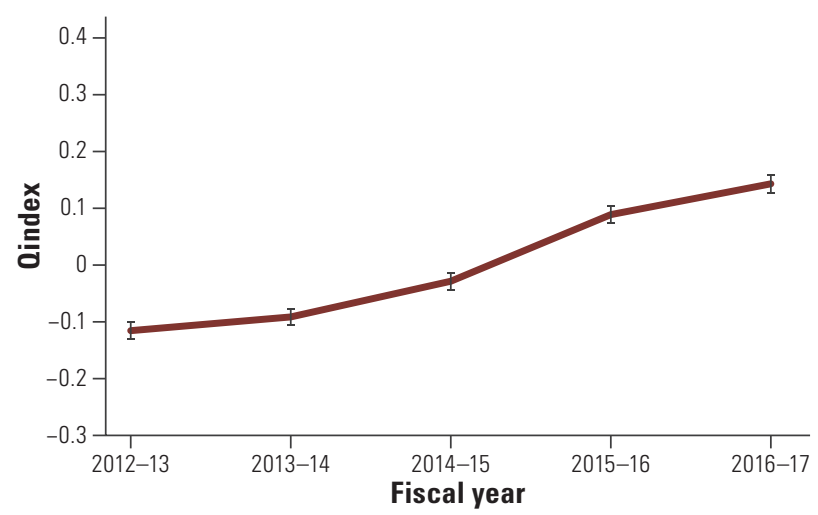

Note: Error bars represent the standard error.

\section{Qindex by home size}

There was a significant main effect of home size, $F(2,601)=13.59$, partial $\eta^{2}=0.04, p<0.001$. Follow-up analyses revealed that small homes had a significantly lower overall Qindex score (mean $[M]=-0.09, S D=0.34$ ) year over year compared to medium $(M=0.05, S D=0.33)$ and large homes $(M=0.05$,
$S D=0.34), p s<0.001$ (there was no significant difference between medium and large homes). There was also a significant home size $\times$ year interaction, $F(6.04,1814.35)=2.307$, partial $\eta^{2}=0.01, p=0.032$. Small homes started off with lower Qindex scores (in 2012/2013) and did not catch up with their larger peers during the five-year period (Figure 2).

\section{Qindex by operator size}

With respect to Qindex by operator size, the results revealed a significant main effect of operator size, $F(2,417)=9.45$, partial $\eta^{2}=0.04, p<0.001$. There was also a significant operator size $\times$ year interaction, $F(6.20,1293.57)=3.51$, partial $\eta^{2}=0.02, p=0.002$. Follow-up analyses revealed that large operators were doing better on overall quality performance $(M=0.11, S D=0.29)$ than medium $(M=-0.01, S D=0.31)$ and small $(M=-0.04, S D=0.34)$ operators, $p \leq 0.001$ (with no significant difference between medium and small operators). In addition, the Qindex scores for large homes appeared to increase at a greater rate than those for medium and small operators (Figure 2).

\section{Qindex by ownership}

With respect to ownership, the results revealed a significant main effect of ownership, $F(2,601)=9.85$, partial $\eta^{2}=0.03, p<0.001$. 
Follow-up analyses revealed that municipal homes performed lower on overall quality $(M=-0.13, S D=0.32)$ than private $(M=0.02, S D=0.32)$ and not-for-profit $(M=0.04, S D=0.39)$ homes, $p s<0.001$ (there was no significant difference between private and not-for-profit homes). In addition, there was no significant ownership $\times$ year interaction, $F(6.02,1810.15)=1.52$, partial $\eta^{2}=.01, p=.167$ (Figure 2).

\section{FIGURE 2.}

Qindex by a) home size, b) operator size and c) ownership

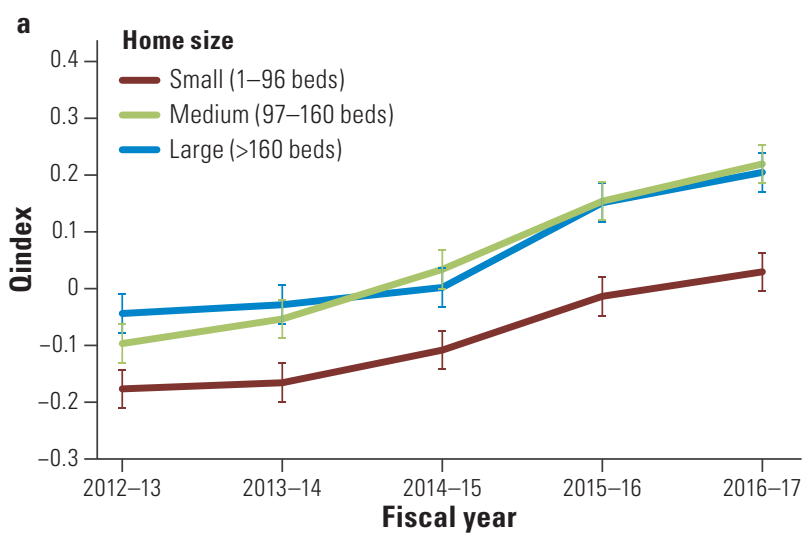

b

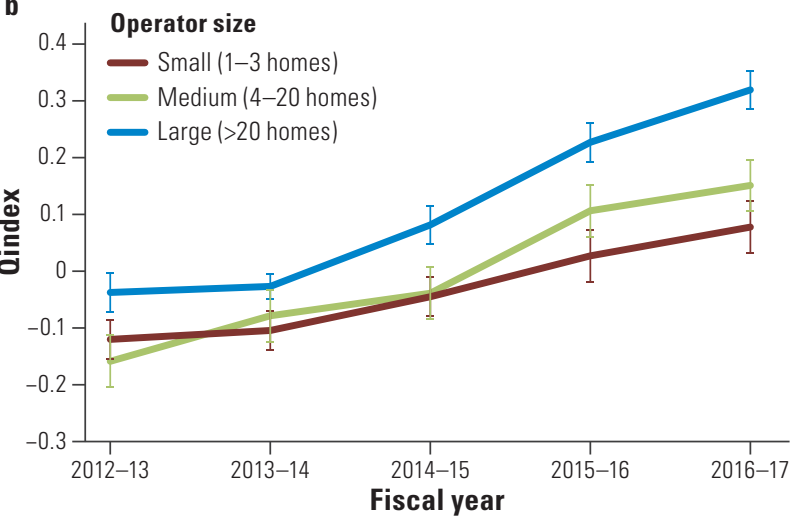

c

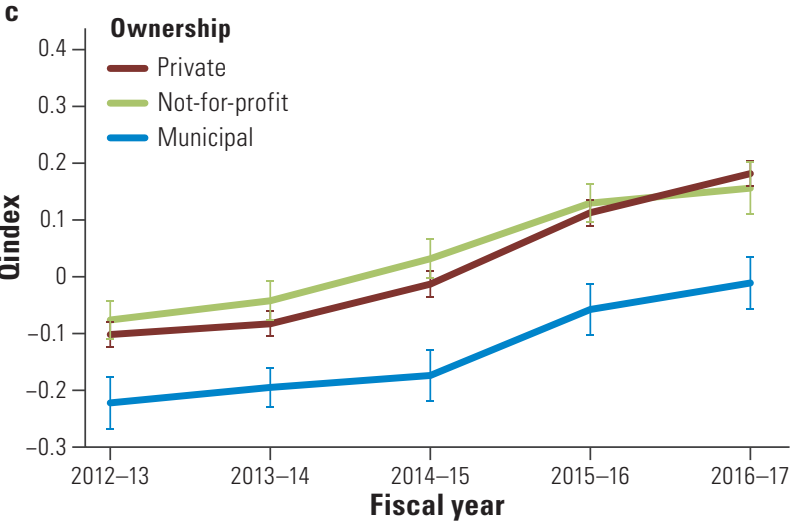

Note: Only members of the Ontario Long Term Care Association (OLTCA) are included in the graph related to operator size. Error bars represent the standard error.

\section{Discussion}

Maintaining public confidence in the LTC system is of critical importance. Overall, there is a robust finding that sector-wide quality performance is showing significant improvement over a five-year period (2012-2017), as indicated by the Qindex. Our analyses also suggest that several organizational-level factors may influence quality performance in LTC homes.

In particular, it was established that not-for-profit and private homes perform similarly on quality. This is contrary to some review studies that have reported better results for not-forprofit versus for-profit (private) homes (Comondore et al. 2009; Hillmer et al. 2005; McGregor et al. 2006; Tanuseputro et al. 2015; You et al. 2016). This discrepancy is likely because of the use of heterogeneous and non-standardized measures of quality in the literature - such as resident comfort, autonomy and security; home-like environment, privacy and dignity; hospitalization, mortality and infection prevalence; and staffing and pressure injuries - rather than the consistent set of nine performance measures (QIs) covered by the Qindex. The present work is the first to assess changes in a standardized and global measure of quality across the entire LTC sector in Ontario.

The results also revealed that at least one type of publicly operated home, for example, municipal operators, may be lagging - rather than leading - in overall quality performance. Although previous research did not distinguish between municipal and not-for-profit homes (Tanuseputro et al. 2015), the current findings indicate that not-for-profit and municipal homes should be separated in terms of ownership type, when examining differences in quality performance.

The findings also suggest that small- and medium-sized operators are not doing as well as large operators in terms of overall quality performance. This may be because larger operators have better access to resources, such as corporate support structures and economies of scale. Perhaps because of increased scalability, larger chain organizations (e.g., large operators) were found to be more likely to integrate new innovations (Castle 2001) - such as computerized medical records or flooring to minimize injury following a fall - which may ultimately have a positive impact on quality-of-care outcomes. In addition, large operators may have a quality performance advantage because they implement systematic management, with greater monitoring of quality outcomes. Support in favour of this view comes from reports that some large operators have created internal algorithms for monitoring quality performance and for identifying homes that need targeted quality improvement interventions. Further research is needed to evaluate implementation of various quality improvement strategies, such as innovation uptake, seeking management services or joining a broader quality consortium.

Finally, the presented findings reveal that homes with a small number of beds (1-96) appear to be having a harder time achieving the same level of quality performance 
compared to medium-sized (97-160 beds) and large (160+ beds) homes. One possible explanation for this finding is that organizational factors, such as home size, may influence a home's capacity to innovate. This is in line with Castle (2001), who showed that larger-bed-size homes were most likely to be in the first $20 \%$ of homes to integrate new innovations.

In contrast to our results, Baldwin et al. (2017) suggested that small home size positively impacted quality performance. This discrepancy may be because the data examined in Baldwin et al. were generated from various countries (e.g., Canada, US, Australia, UK and the Netherlands), with the majority being from the US. Interjurisdictional comparisons in the LTC sector must be done with caution because of fundamental differences in how LTC is provided across countries (e.g., funding) and inconsistencies in quality measurements studied.

In our view, findings based on the Qindex are more reliable than reviews such as those from Comondore et al. (2009) or Baldwin et al. (2017) because the Qindex uses a single global measure of quality instead of a wide variety of indicators. Specifically, Comondore et al. (2009) used indicators of service quality (e.g., behaviours, falls, infections, mortality, quality of life, pressure injuries), care (e.g., hospital use, staffing hours) and regulatory compliance (e.g., complaints), as well as composite indicators. Given the varying definitions and measures of quality, it is unsurprising that our Qindex findings diverge from those reported in earlier literature. Although the composition of QIs used in the Qindex could be refined, it is representative of quality measures routinely collected in the Canadian LTC sector (across all provinces). As demonstrated in this paper, the Qindex shows promise as a tool for measuring overall quality - with a focus on trends over time and comparisons between homes and types of homes.

\section{The Qindex is a potentially valuable metric to help describe quality improvement across the entire LTC sector.}

\section{Strengths and limitations Strengths}

Across Canada, data on quality performance are publicly reported and available annually. This ensures that data analyses using the Qindex can continuously be updated and refreshed, year over year. The use of InterRAI data, as the inputs to the metric, also allows for future work to assess quality of integrated health systems by incorporating data from service providers accessed over the entire patient journey. In addition, the data are consistently and systematically collected in the LTC sector across Canada, avoiding potential confounding because of inconsistencies in rating methodology.

\section{Limitations}

Potential limitations associated with the Qindex are related to the composition of the score itself. Because $\mathrm{z}$ scores are used, variables with low variability may have a disproportionate effect on Qindex scores. In addition, operator size data were pulled from the OLTCA's internal membership database; therefore, only members of the OLTCA were included in the analyses and figure related to "operator size." Note that the differences and trends in Qindex reported in this paper, although statistically significant, are relatively modest, being around 0.2 , or 0.25 of a standard deviation unit. Last, the Qindex only includes clinical, quality-of-care outcomes. There are no quality-of-life measures, such as resident experience or family satisfaction, included in the score. As such, the voices of the residents and their families are not measured by the Qindex. Further issues associated with the development and use of the Qindex are discussed elsewhere (Wilkinson et al. 2019).

\section{Conclusion}

The Qindex has been demonstrated as a composite score that can be used to assess changes in LTC quality over time and between various types of homes. The Qindex is a potentially valuable metric to help describe quality improvement across the entire LTC sector. Global metrics of quality, such as the Qindex, are necessary tools for quality management. Only by effectively measuring and managing quality will it be possible to ensure that all individuals residing in LTC homes - in a jurisdiction such as Ontario - can get the type of care they need and deserve. Furthermore, in the context of the recently announced Ontario Health and the emphasis on improving integration, global metrics should prove even more valuable to assess the overall performance of a health system over a person's journey that may include multiple service providers.

This paper showed that, from 2012 to 2017, Ontario LTC homes demonstrated continuous improvements in overall quality. The findings reported herein also supplement the research literature by showing how quality comparisons between different types of homes can be made using a consistent, global measure of quality. HQ

\section{Acknowledgement}

This research was funded by the Canadian Institutes of Health Research (CIHR) Health System Impact Fellowship (HSIF) awarded to the first author, the Ontario Long Term Care Association and the University of Toronto.

\section{References}

Baker, G.R., P.G. Norton, V. Flintoft, R. Blais, A. Brown, J. Cox et al. 2004. "The Canadian Adverse Events Study: The Incidence of Adverse Events Among Hospital Patients in Canada." CMAJ 170(11): 1678-86. doi:10.1503/cmaj.1040498. 
Baldwin, R., L. Chenoweth, M. dela Rama and A.Y. Wang. 2017. "Does Size Matter in Aged Care Facilities? A Literature Review of the Relationship Between the Number of Facility Beds and Quality." Health Care Management Review 42(4): 315-27. doi:10.1097/ HMR.0000000000000116.

Berta, W., A. Laporte and W.P. Wodchis. 2014. "Approaches to Accountability in Long-Term Care." Healthcare Policy 10(Special Issue): 132-44.

Canadian Institute for Health Information (CIHI). 2016. CIHI's Strategic Plan, 2016 to 2021. Retrieved September 19, 2018. <https:// www.cihi.ca/en/strategicplan2016-2021-enweb.pdf>.

Canadian Institute for Health Information (CIHI). 2016-2017. Continuing Care Reporting System (CCRS). Retrieved September 14, 2018. <https://www.cihi.ca/en/continuing-care-metadata>.

Canadian Institute for Health Information (CIHI). 2017. Provincial/ Territorial Data Quality Report: Indicators and Contextual Measures. Ottawa, ON: Author.

Canadian Institute for Health Information (CIHI). 2019a. Canadian Institute for Health Information (CIHI). Retrieved January 7, 2019. $<$ www.cihi.ca>.

Canadian Institute for Health Information (CIHI). 2019b. Canadian Institute for Health Information's (CIHI) Your Health System. Retrieved January 7, 2019. <https://yourhealthsystem.cihi.ca>.

Castle, N.G. 2001. "Innovation in Nursing Homes: Which Facilities Are the Early Adopters?" Gerontologist 41(2): 161-72.

Comondore, V.R., P.J. Devereaux, Q. Zhou, S.B. Stone, J.W. Busse, N.C. Ravindran et al. 2009. "Quality of Care in For-Profit and Not-for-Profit Nursing Homes: Systematic Review and Meta-Analysis." BMJ 339: b2732. doi:10.1136/bmj.b2732.

Deber, R.B. 2014. “Thinking About Accountability.” Healthcare Policy 10(Special Issue): 12-24. doi:10.12927/hcpol.2014.23932.

Denis, J.-L. 2014. "Accountability in Healthcare Organizations and Systems." Healthcare Policy 10(Special Issue): 8-11. doi:10.12927/ hcpol.2014.23933.

Eliasoph, H., B. Monaghan, R. Beaudoin, R. Cushman, G. DuBoisWing, M.J. Emery et al. 2007. "'We Are All in This Together': Integrated Health Service Plans in Ontario." Healthcare Quarterly 10(3): 82-90. doi:10.12927/hcq.2007.18938.

Field, A., J. Miles and Z. Field. 2012. Discovering Statistics Using R. London, UK: Sage Publications.

Health Quality Ontario (HQO). 2013a. Common Quality Agenda: An Overview. Retrieved August 29, 2018. <http://www.hqontario.ca/portals/0/ documents/home/cqa-01-common-quality-agenda-powerpoint.pdfs.

Health Quality Ontario (HQO). 2013b. Long-Term Care Restraint Indicator Informational Webinar. Retrieved March 22, 2019. <http:// www.hqontario.ca/Portals/0/Documents/pr/ltc-restraint-indicatorinformation-webinar-slide-deck-en.pdf>.

Health Quality Ontario (HQO). 2014. Monitoring What Matters: Health Quality Ontario's Approach to Performance Monitoring and Public Reporting. Retrieved August 29, 2018. <http://www.hqontario. $\mathrm{ca} /$ Portals/0/documents/pr/monitoring-what-matters-en-1410.pdf>.

Health Quality Ontario (HQO). 2015. LTC Indicator Review Report: The Review and Selection of Indicators for Long-Term Care Public Reporting. Retrieved March 22, 2019. <https://www.hqontario. $\mathrm{ca} /$ Portals/0/documents/system-performance/ltc-indicator-reviewreport-november-2015.pdf>.
Health Quality Ontario (HQO). n.d. "Long-Term Care Home Performance in Ontario." Retrieved August 29, 2018. <http:// www.hqontario.ca/System-Performance/Long-Term-Care-HomePerformances.

Hillmer, M.P., W.P. Wodchis, S.S. Gill, G.M. Andreson and P.A. Rochon. 2005. "Nursing Home Profit Status and Quality of Care: Is There Any Evidence of an Association?" Medical Care Research and Review 62(2): 139-66.

Kromm, S.K., G.R. Baker, W.P. Wodchis and R.B. Deber. 2014. "Acute Care Hospitals' Accountability to Provincial Funders." Healthcare Policy 10(Special Issue): 25-35. doi:10.12927/hcpol.2014.23852.

McGregor, M.J., R.B. Tate, K.M. McGrail, L.A. Ronald, A.-M. Broemeling and M. Cohen. 2006. "Care Outcomes in Long-Term Care Facilities in British Columbia, Canada: Does Ownership Matter?" Medical Care 44(10): 929-35.

Mukamel, D.B., D.L. Weimer, W.D. Spector, H. Ladd and J.S. Zinn. 2008. "Publication of Quality Report Cards and Trends in Reported Quality Measures in Nursing Homes." Health Services Research 43(4): 1244-62. doi:10.1111/j.1475-6773.2007.00829.x.

Mukamel, D.B., Z. Ye, L.G. Glance and Y. Li. 2015. "Does Mandating Nursing Home Participation in Quality Reporting Make a Difference? Evidence from Massachusetts." Medical Care 53(8): 713-19. doi:10.1097/MLR.0000000000000390.

Ontario Long Term Care Association. 2018. This Is Long-Term Care 2018. Retrieved August 3, 2018. <https:/www.oltca.com/OLTCA/ Documents/Reports/ThisIsLongTermCare2018.pdf>.

Ontario Ministry of Health and Long-Term Care (MOHLTC). 2008. "Long-Term Care Home Quality Inspection Program." Retrieved January 7, 2019. <http://www.health.gov.on.ca/en/public/programs/ ltc/31_pr_inspections.aspx $>$.

Ontario Ministry of Health and Long-Term Care (MOHLTC). 2019. "Ontario Ministry of Health and Long-Term Care's (MOHLTC) Reports on Long-Term Care Homes." Retrieved January 7, 2019. $<$ http://publicreporting.ltchomes.net/en-ca/default.aspx>.

Tanuseputro, P., M. Chalifoux, C. Bennett, A. Gruneir, S.E. Bronskill, P. Walker et al. 2015. "Hospitalization and Mortality Rates in Long-Term Care Facilities: Does For-Profit Status Matter?" Journal of the American Medical Directors Association 16(10): 874-83. doi:10.1016/j.jamda.2015.06.004.

Wilkinson, A., V. Haroun, T. Wong, N. Cooper and M. Chignell. 2019. Qindex: Tracking Quality in Long-Term Care. Manuscript in preparation.

You, K., Y. Li, O. Intrator, D. Stevenson, R. Hirth, D. Grabowski et al. 2016. "Do Nursing Home Chain Size and Proprietary Status Affect Experiences with Care?" Medical Care 54(3): 229-34. doi:10.1097/ MLR.0000000000000479.

\section{About the Authors}

Andrea Wilkinson, $\mathrm{PhD}$, is a research assistant in the Department of Mechanical and Industrial Engineering, University of Toronto. Dr. Wilkinson's research focuses on evaluating and enhancing quality of care and well-being for residents living in long-term care homes, their families and long-term care staff. 
Vinita Haroun, MSc, HBSc, is the director of the Centre for Research and Innovation Support, University of Toronto. At the time of this study, Ms. Haroun provided strategic leadership at the OLTCA for research and innovation initiatives as their director of research and knowledge translation, with the goal of supporting long-term care operators to provide safe, high-quality care and excellent quality of life for residents.

Tommy Wong, BComm, is the director of data and analytics, Ontario Long Term Care Association. As director, Mr. Wong provides leadership to the association's data infrastructure, develops business intelligence solutions and conducts analyses with several key long-term care data sources to help improve the sector's key priorities.
Nancy Cooper, BSc, MHSA, is the director of quality and performance, Ontario Long Term Care Association. Ms. Cooper provides strategic leadership to the association's monitoring, analysis and evaluation of provincial regulatory and public policy initiatives that impact sector quality and performance outcomes with the goal of informing current and future directions in public policy. She is adjunct professor, University of Toronto, Institute of Health Policy, Management and Evaluation.

Mark Chignell, PhD, is a professor in the Department of Mechanical and Industrial Engineering, University of Toronto. Dr. Chignell carries out research in human factors and user interface design, with an interest in aging, adaptive interfaces and healthcare. His research is supported by AGE-WELL NCE Inc., a member of the Networks of Centres of Excellence program.

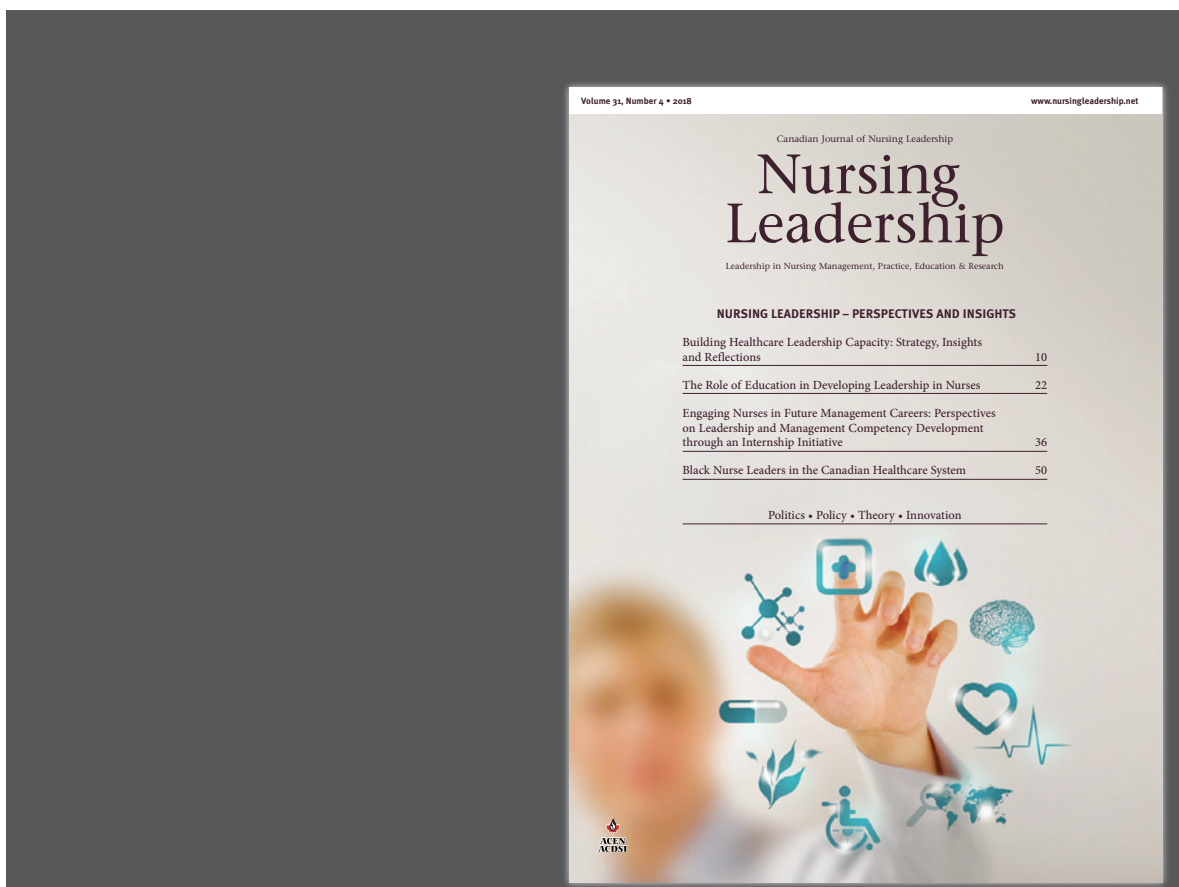

NursingLeadership.net 